\title{
Introducción de un portafolio para fomentar competencias transversales de los estudiantes universitarios*
}

Use of a portfolio to improve generic skills of the pre-graduate students.

Mireia Valero, José Aramburu, Josep-Eladi Baños, Mariano Sentí y Jorge Pérez

Facultad de Ciencias de la Salud y de la Vida. Universidad Pompeu Fabra (Barcelona)

Durante el curso 2003-04, en la Facultad de Ciencias de la Salud y de la Vida de la Universidad Pompeu Fabra se inició la experiencia de introducir un portafolio para fomentar cuatro competencias transversales relevantes: comunicación oral delante de un auditorio, comunicación escrita, búsqueda de información mediante el uso de las nuevas tecnologías y trabajo en equipo. La experiencia se inició con los estudiantes del último curso de los estudios de Biología, que tienen una orientación biosanitária. Después de una reacción contraria inicial por parte de los estudiantes, el portafolio final propuesto fue semiestructurado, tutorizado por un profesor $y$ de realización voluntaria. La evaluación fue dicotómica,

* La experiencia descrita en este artículo recibió una ayuda del programa de Mejora de la Calidad Docente universitaria (MQD) de 2003 de la Generalitat de Cataluña.

Correspondencia:

Mireia Valero

Facultad de Ciencias de la Salud y de la Vida

Universidad Pompeu Fabra

c/Dr. Aiguader, 80, 08003 Barcelona

tel. 935422833

e-mail: mireiavm@teleline.es positiva o negativa. Si se realizaba la actividad, el estudiante obtenía un plus sobre la nota en una asignatura con un número elevado de créditos. Cerca del 50 \% de los alumnos participó en el primer año y el $75 \%$ en el segundo. Todos los participantes superaron el ejercicio. La dedicación media invertida por los estudiantes en su realización fue aproximadamente 15 horas y los tutores prestaron una dedicación media de $\mathbf{2}$ horas en la actividad. La mayoría de participantes, estudiantes y tutores, manifestaron satisfacción con la actividad, siendo más elevada en los tutores.

La experiencia ha sido valorada muy positivamente y ha servido para generalizarla a todos los alumnos e iniciarla desde el primer curso de carrera.

Palabras clave: Portafolio. Competencias transversales. Autoaprendizaje. Métodos de evaluación.

In the 2003-4 academic year, the Faculty of Health and Life Sciences of the Pompeu Fabra University launched the experience of introducing a portfolio to encourage interest in developing four relevant generic skills: public presentation skills, written communication, the ability to search for information using new technologies, and teamwork. The first 
participants of the experience were final year students of Biology. This career emphasizes in human biology and has a biosanitary orientation.

After an initial negative reaction from the students, the final portfolio as presented was semi-structured, voluntary, and tutored by a university lecturer. Only two possible evaluations were used - positive or negative. If a student carried out the activity, he or she obtained a "plus" on top of the mark for a subject with a high number of credits.

Approximately $\mathbf{5 0} \%$ of the students participated in the first year and $75 \%$ in the second. All of the participants passed the exercise, with the students dedicating an average of $\mathbf{1 5}$ hours to the activity and the tutors an average of 2 hours. Most of the participants, both students and tutors, declared their satisfaction with the activity, with the tutors reporting a higher rate of satisfaction than the students.

The evaluation of the experience was very positive and as a result the activity has now become a general one for all students from the first year of their university studies.

Key words: Portfolio. Generic Skills. Self-instruction program. Educational assessment.

\section{INTRODUCCIÓN}

Las universidades europeas se encuentran inmersas en un proceso de cambio derivado de las orientaciones surgidas en la convergencia al espacio europeo de enseñanza superior (EEES). Dicha convergencia universitaria implica cambios en la organización de los estudios y, fundamentalmente, cambios metodológicos en los procesos educativos. Desde el punto de vista metodológico destaca la necesidad de desarrollar una enseñanza centrada en quien aprende, establecer el aprendizaje en un marco definido en competencias y modificar el rol del profesor. En este nuevo contexto surge como prioritaria la necesidad de fomentar las competencias transversales de los estudiantes y de introducir nuevos instrumentos para potenciar y evaluar su aprendizaje.

El portafolio es una herramienta docente relativamente nueva que se está utilizando con éxito para el fomento en los alumnos de habilidades de autoapren- dizaje y para la evaluación del nivel competencial alcanzado ${ }^{1-3}$. Es un instrumento que permite a cualquier persona reflexionar sobre una actividad determinada para mejorarla y, también, permite acreditar su dominio. Para los expertos, la dinámica central del portafolio sería la reflexión sobre la práctica realizada con la intención de mejorarla ${ }^{1,4,5}$ y la actividad debería ser llevada a cabo de forma tutorizada ${ }^{6,7}$. La introducción del ejercicio sería especialmente idónea para la capacitación y evaluación del alumno en competencias transversales o genéricas ${ }^{8}$.

El uso de portafolios está siendo recomendado por las autoridades educativas de nuestro entorno ${ }^{9} \mathrm{y}$, en el campo de ciencias de la salud, se conocen experiencias muy sólidas de implementación del instrumento en las facultades de medicina de las universidades de Dundee en Escocia ${ }^{2,10}$ y de Maastricht en Holanda ${ }^{1,6,11}$.

La Facultad de Ciencias de la Salud y de la Vida (FCSV) de la Universidad Pompeu Fabra (UPF) inició en el curso 1998-99 su actividad con la titulación de Biología. Dichos estudios son de cinco años, están centrados en la biología humana con una orientación exclusivamente biosanitaria, tienen un número reducido de alumnos, 60 por curso, y cuentan con un proyecto educativo innovador ${ }^{12}$.

En la FCSV siempre se ha estimulado el desarrollo de las competencias transversales y desde el primer curso los estudiantes tienen ocasión de practicar, en las distintas asignaturas y en actividades especialmente diseñadas para ello, muchas de las propuestas en el proyecto "Tuning" sobre la convergencia europea de enseñanza superior ${ }^{13}$. De todos modos, y para reforzar lo anterior, hace algunos años los responsables educativos del centro decidieron explicitar como objetivo educativo el fomento y dominio de cuatro competencias genéricas relevantes y muy presentes en la actividad cotidiana de la facultad: hablar en público ante un auditorio, escribir correctamente, buscar información usando las nuevas tecnologías de la información y trabajar en equipo.

Así, en el curso 2003-04 se inició la experiencia de introducción de un portafolio para fomentar el desarrollo de las competencias citadas y la capacidad de acreditar su domino por los estudiantes.

El objetivo de este escrito es narrar el proceso de introducción de este portafolio y reflexionar sobre los resultados de participación, dedicación y satisfacción de los participantes en los dos primeros años de la experiencia. 


\section{DESARROLLO DE LA EXPERIENCIA}

\section{a) Requisitos previos}

Cuando las autoridades educativas decidieron introducir el portafolio, en primer lugar procedimos a determinar el estado de la cuestión. En Marzo de 2003, en las bases de datos Medline y Eric, realizamos una búsqueda bibliográfica exhaustiva sobre experiencias donde se había utilizado el portafolio como instrumento formativo, tanto en la formación pregraduada y postgraduada como en el desarrollo profesional continuado. En el campo de la educación en ciencias de la salud encontramos dos documentos de la Association for Medical Education in Europe (AMEE) que consideramos muy relevantes: Portfoliobased learning and assessment in medical education ${ }^{3}$ y Portfolios as a method of student assessment ${ }^{2}$. Dichos documentos, junto con la publicación del Royal Collage of General Practitioners británico Portfolio-based Learning in General Practice ${ }^{7}$, constituyeron el marco de referencia del nuestro trabajo.

Entre las experiencias de aplicación del portafolio en la formación pregraduada se escogieron las dos que consideramos más importantes, las de las facultades de medicina de las universidades de Dundee (Escocia) y de Maastricht (Holanda). Estas fueron analizadas detenidamente y también contactamos con sus responsables. Paralelamente, las personas que debíamos dirigir la experiencia aumentamos nuestra formación asistiendo a varios talleres monográficos sobre el instrumento.

\section{b) Ubicación en el currículum}

\section{y contingencia sumativa}

Sabiendo que el portafolio era para fomentar las competencias transversales citadas, que debería ser evaluado y que debería tener repercusión acreditativa en el expediente de los estudiantes, el siguiente paso consistió en determinar en qué asignatura del currículum se aplicaría y cual sería su repercusión sobre la nota.

Se consideró que el último curso de carrera era el más idóneo para iniciar la experiencia. También se pensó en que la actividad podría ser ubicada en las asignaturas prácticas de dicho curso. Los estudios de Biología de la FCSV fueron diseñados principalmente para fomentar la inserción profesional de sus graduados. Así, el último curso prevé tres itinerarios profesionales (Industria sanitaria, Investigación biomédica y Laboratorio de análisis) con la realización de un semestre de prácticas profesionalizadas en un entorno laboral autentico. Dicha actividad de prácti- ca real es contemplada en una asignatura de 20 créditos que es evaluada por diversos métodos ${ }^{14}$.

Los responsables de los itinerarios aceptaron la propuesta de la dirección del centro de que la realización del portafolio fuera evaluada como una actividad de la asignatura de prácticas con una contingencia sobre su nota del $10 \%$. Así mismo, estos profesores se convirtieron en agentes activos del proyecto.

\section{c) Tipo de portafolio}

Seguidamente, después de diversas reuniones, los responsables educativos y los implicados en el proyecto decidimos el tipo de portafolio que se quería implementar partiendo de la premisa que sería para desarrollar y acreditar las cuatro competencias transversales citadas, así como, habilidades de autoaprendizaje y de acreditación en general.

Se diseñó un portafolio con los rasgos esenciales del de la Facultad de Medicina de Maastricht ${ }^{1,6}$, se trataba de un portafolio de realización obligatoria, con finalidad formativa y sumativa, semi-estructurado y tutorizado por un profesor.

Siguiendo las recomendaciones más acreditadas se elaboró una manual para los participantes, "La Guía del portafolio para los estudiantes de quinto"15, en la que, alumnos y tutores, tenían toda la información sobre el instrumento y la dinámica propuesta para su realización. La guía constaba de cinco apartados: 1) Definición del instrumento, 2) Estructura del mismo, 3) Soporte para su elaboración, 4) Evaluación de la actividad y 5) Directrices para su elaboración. En un anexo se facilitaba una plantilla de análisis para cada competencia, estructurada en puntos fuertes, puntos débiles, sugerencias para la mejora y actividades en las que se intentaría la mejora.

Los estudiantes deberían tener dos reuniones con sus tutores. La primera, tras una reflexión previa de los alumnos rellenando las plantillas del anexo, versaría sobre puntos fuertes, puntos débiles y propuestas concretas de mejora en cada una de las cuatro competencias. Al finalizar esta reunión, las plantillas complementadas serían firmadas por el estudiante y por el tutor. En la segunda reunión se comentaba el contenido que tendría el portafolio. Así mismo, al finalizar el curso, los estudiantes deberían presentar un portafolio que incluyera, para cada competencia, la plantilla firmada de la primera reunión, un escrito de reflexión en un único folio sobre la adquisición y dominio de ésta y, finalmente, tres o más documentos para acreditar lo que se manifestaba en el escrito. 


\section{d) Información a los estudiantes,} reacción de éstos y ajuste de la actividad.

Al comenzar el curso académico los estudiantes fueron informados del inicio de la actividad anunciándoles que recibirían información posterior más precisa. Así, una vez elaborada la guía, ésta se distribuyó por correo electrónico y se convoco a los alumnos a una sesión donde recibirían información exhaustiva sobre el ejercicio.

Inmediatamente después de la distribución de la guía y previamente a la realización de la sesión informativa prevista, los estudiantes iniciaron manifestaciones contrarias a la realización de la actividad y, principalmente, opuestas a que ésta fuera obligatoria. El alumnado mostró su rechazo con una carta dirigida a la dirección del centro así como con carteles y pancartas en los espacios de la facultad.

Ante tal reacción, los responsables del proyecto advertimos que se había malinterpretado el valor educativo del ejercicio y asumimos errores en el proceso de información a los estudiantes. Después de analizar la situación con la dirección del centro y con los responsables de los itinerarios, procedimos a realizar ligeras modificaciones para optimizar la actividad. Consideramos que ésta fuera voluntaria, si bien manteniendo una contingencia sobre la nota de la asignatura. A su vez, se simplificó la evaluación del ejercicio optándose por una calificación dicotómica de superado/no-superado, según el cumplimiento de unos requisitos mínimos. Los estudiantes que realizaran y superaran el ejercicio tendrían un plus de 0,5 puntos sobre la nota de la asignatura de prácticas del itinerario.

A partir de este momento, la experiencia se desarrolló con normalidad durante el primer curso de aplicación. Durante el segundo curso no se registró ningún tipo de incidente y la actividad fue realizada con total normalidad desde el principio.

Cada estudiante participante debía tener un tutor. Dado que en la FCSV existía una programa voluntario de tutorías personalizadas con muchos alumnos y profesores implicados ${ }^{16}$, aquellos estudiantes que ya tuvieran tutor serían tutorizados por éste. Los responsables del proyecto (y autores de este escrito) ejercieron de tutores del portafolio de los alumnos que no tenían tutor hasta el momento.

\section{RESULTADOS DE LA EXPERIENCIA}

\section{a) Participación y resultados académicos}

Respecto a la participación en el ejercicio, durante el curso 2003-04 realizaron la actividad 26 alumnos, el $45,6 \%$ de los matriculados, 16 mujeres ( $61,5 \%$ ) y 10 hombres $(38,5 \%)$. En el segundo año, curso 200405, participaron 41 estudiantes, el 74,5\%, 36 mujeres $(87,8)$ y 5 hombres $(12,2 \%)$. El $100 \%$ de los profesores que eran tutores de alumnos participantes tomaron parte en la actividad, 11 el primer año y 17 el segundo.

A partir de los criterios marcados, todos los estudiantes participantes superaron la actividad y, por tanto, obtuvieron el plus previsto sobre la nota de prácticas.

\section{b) Dedicación}

Los participantes, estudiantes y tutores, debían registrar el tiempo de dedicación a la actividad y facilitar dicha información a los responsables del proyecto. La tabla 1 presenta el tiempo invertido por los alumnos en la actividad. Se puede comprobar que el tiempo dedicado por éstos a las diferentes partes del ejercicio fue muy parecido en los dos cursos.

La tabla 2 describe el tiempo invertido en la actividad por los tutores. El tiempo total medio dedicado por el tutor a un estudiante en la realización del ejercicio fue aproximadamente de 2,3 horas en el curso 2003-04 y de 1,8 horas el curso 2004-05.

Considerando las dos tablas ( 1 y 2 ), se puede estimar que los estudiantes destinaron unas 14,3 horas durante el curso 2003-04 y unas 14,9 horas durante el curso 2004-05 para realizar la actividad del portafolio.

Tabla 1. Tiempo invertido por los estudiantes, expresado en horas, en la realización de la actividad

\begin{tabular}{|c|c|c|c|}
\hline Tempo / hora & & $2003-2004$ & $2004-2005$ \\
\hline $\begin{array}{l}\text { Preparación } \\
1^{\text {a }} \text { reunió }\end{array}$ & $\begin{array}{l}\text { Media } \\
\text { D.típica } \\
\text { Moda } \\
\text { Rango }\end{array}$ & $\begin{array}{c}3,5 \\
2,2 \\
2 \\
1-9\end{array}$ & $\begin{array}{c}3 \\
1,9 \\
2 \\
1-10\end{array}$ \\
\hline $\begin{array}{l}\text { Preparación } \\
2^{\mathrm{a}} \text { reunió }\end{array}$ & $\begin{array}{l}\text { Media } \\
\text { D.típica } \\
\text { Moda } \\
\text { Rango }\end{array}$ & $\begin{array}{c}4,9 \\
1,8 \\
4 \text { i } 6 \\
1,5-8\end{array}$ & $\begin{array}{c}6,3 \\
4,1 \\
3 \text { i } 5 \\
1-15\end{array}$ \\
\hline $\begin{array}{l}\text { Elaboración } \\
\text { del porta- } \\
\text { folio }\end{array}$ & $\begin{array}{l}\text { Media } \\
\text { D.típica } \\
\text { Moda } \\
\text { Rango }\end{array}$ & $\begin{array}{c}3,5 \\
4,2 \\
2 \\
0-10\end{array}$ & $\begin{array}{c}3,8 \\
3,2 \\
0,2 \text { i } 3 \\
0-13\end{array}$ \\
\hline TOTAL* & $\begin{array}{l}\text { Media } \\
\text { D.típica } \\
\text { Moda } \\
\text { Rango }\end{array}$ & $\begin{array}{c}12 \\
4,6 \\
15 \\
4-25\end{array}$ & $\begin{array}{c}13,1 \\
6,3 \\
15 \text { i } 20 \\
4-30\end{array}$ \\
\hline
\end{tabular}

* Tiempo total de realización de la actividad, sin el tiempo dedicado a las reuniones. 
Tabla 2. Tiempo invertido por los tutores, expresado en horas, en la realización de la actividad

\begin{tabular}{c|l|c|c}
\hline \multicolumn{2}{l|}{ Tempo / horas } & 2003 - 2004 & 2004 - 2005 \\
\hline \multirow{4}{*}{$1^{\text {a }}$ reunión } & Media & 1,3 & 1 \\
& D.típica & 0,7 & 0,3 \\
& Moda & 1 & 1 \\
& Rango & $0.5-3.5$ & $0.42-2$ \\
& & & \\
& Media & 1 & 0,8 \\
2a reunión & D.típica & 0,6 & 0,3 \\
& Moda & 1,5 & 1 \\
& Rango & $0.08-2$ & $0.33-1.67$ \\
\hline
\end{tabular}

\section{c) Satisfacción de los participantes}

Al finalizar el ejercicio, los participantes, tanto estudiantes como tutores, contestaron una encuesta anónima en la que mostraban, en una escala de cinco alternativas (desde "muy baja" a "muy alta"), el grado de satisfacción con la experiencia.

Contestaron la encuesta de satisfacción 18 de los 26 $(69,2 \%)$ estudiantes que realizaron el portafolio en el curso 2003-04 y 33 de 41 (80,5\%) en el curso 2004-05. Respecto a los tutores, contestaron la encuesta 9 de los $11(81,8 \%)$ participantes en el primer año y los 17 (100\%) participantes del segundo.

Las tablas 3 y 4 muestran los resultados de satisfacción de estudiantes y tutores respectivamente.

La actitud general de los alumnos con la experiencia fue positiva ya que mostraron satisfacción más de un $70 \%$ de los participantes en el primer año y más de un $80 \%$ en el segundo.

Respecto a los tutores, la actividad fue valorada positivamente por más del $85 \%$ de los mismos en los dos años, predominando los valores más altos en la escala de satisfacción.

\section{REFLEXIONES SOBRE LA EXPERIENCIA}

Como se ha expuesto anteriormente, al inicio de la experiencia surgió un conflicto protagonizado por los estudiantes en desacuerdo con la realización obligatoria y evaluada del ejercicio. Los autores creemos que el motivo principal de este hecho se debería a un problema en el proceso seguido en la información a los alumnos. La distribución de la guía antes de llevarse a cabo la sesión informativa exhaustiva sobre la actividad creemos que fue un error de nuestra parte. Se sabe que los estudiantes son propensos a presentar ansiedad cuando se introducen nuevos métodos de evaluación ${ }^{2,17,18}$. En el caso que nos ocupa, creemos que la dinámica de las evaluaciones formativas y sobretodo formadoras 19 de detección de puntos débiles en la actuación realizada para proceder a la planificación de mejoras, sorprendió a los estudiantes y desencadenó la reacción contraria a la realización obligada y evaluada del ejercicio. En general, los estudiantes están habituados a mostrar lo mejor de ellos mismos y no a exponer sus puntos débiles.

La reacción inicial contraria de los alumnos a la introducción del portafolio con repercusión sumativa ha sido descrita también en otras experiencias ${ }^{20}$. Así mismo, es frecuente que los responsables educativos den por hecho que la relación entre el currículum, los procesos docentes y los tipos de evaluación, que ellos tienen tan clara está también asumida por los estudiantes ${ }^{18}$.

El conflicto y la asunción de errores en la planifi-

Tabla 3. Frecuencia y, entre paréntesis, porcentaje, de estudiantes en la valoración del portafolio en los dos cursos.

\begin{tabular}{|c|c|c|c|c|c|}
\hline $\begin{array}{c}\text { Satisfacción general } \\
\text { con el portafolio }\end{array}$ & Muy baja & Baja & Aceptable & Alta & Muy alta \\
\hline $\begin{array}{c}2003-04 \\
N=18\end{array}$ & $2(11,1)$ & $3(16,6)$ & $\mathbf{8}(\mathbf{4 4 , 4 )}$ & $\mathbf{4 ( 2 2 , 2 )}$ & $\mathbf{1 ( 5 , 5 )}$ \\
\hline $\begin{array}{c}2004-05 \\
\mathrm{~N}=33\end{array}$ & $2(6,1)$ & $3(9,1)$ & $\mathbf{2 0 ( 6 0 , 6 )}$ & $\mathbf{8 ( 2 4 , 3 )}$ & $\mathbf{0 ( 0 , 0 )}$ \\
\hline
\end{tabular}

Tabla 4. Frecuencia y, entre paréntesis, porcentaje, de tutores en la valoración del portafolio en los dos cursos.

\begin{tabular}{|c|c|c|c|c|c|}
\hline $\begin{array}{c}\text { Satisfacción general } \\
\text { con el portafolio }\end{array}$ & Muy baja & Baja & Aceptable & Alta & Muy alta \\
\hline $\begin{array}{c}2003-04 \\
\mathrm{~N}=9\end{array}$ & $0(0,0)$ & $1(11,1)$ & $\mathbf{0 ( 0 , 0 )}$ & $\mathbf{6}(\mathbf{6 6 , 6 )}$ \\
\hline $\begin{array}{c}2004-05 \\
\mathrm{~N}=17\end{array}$ & $0(0,0)$ & $2(11,8)$ & $\mathbf{5 ( 2 9 , 4 )}$ & $\mathbf{5 ( 2 9 , 4 )}$ & $\mathbf{5 ( 2 9 , 4 )}$ \\
\hline
\end{tabular}


cación de la actividad llevaron a la dirección de la FCSV a reconsiderar la cuestión y a proponer las modificaciones que hemos comentado anteriormente. Creemos que las decisiones de voluntariedad y de simplificación de la evaluación (dicotómica: superado/no superado y con un plus de 0,5 puntos) fueron un acierto sobrevenido. La adopción de una calificación sencilla cualitativa (superado/no superado) facilitó, a su vez, el inicio de estudios piloto sobre objetividad en la evaluación del portafolio que servirían para decidir sobre una posible evaluación cuantitativa en el futuro. Consideramos que estos estudios serían especialmente oportunos dada la escasez de trabajos empíricos sobre objetividad en la evaluación sumativa del portafolio ${ }^{21-24}$.

Creemos que la evaluación de un ejercicio como el que comentamos tiene que tener una contingencia sumativa. Es una premisa universalmente aceptada que el tipo de evaluación de los aprendizajes condiciona el modo de aprender de los estudiantes ${ }^{25-27}$ y se han descrito experiencias frustradas en la introducción del portafolio cuando el ejercicio es propuesto como actividad exclusivamente formativa sin ninguna repercusión sumativa ${ }^{28,29}$.

Nuestra experiencia refuerza la opinión de los expertos de planificar cuidadosamente la introducción del portafolio como actividad evaluada. Es altamente recomendable dispensar una explicación clara a les estudiantes sobre la finalidad educativa del ejercicio, la dinámica propuesta para su realización y como se realizará la evaluación.

La implicación des de la fase de planificación de la actividad de todos los estamentos que de alguna manera estén involucrados, y sobretodo de los estudiantes, es deseable para garantizar la viabilidad del proyecto $^{2,3,30,31}$. Por otro lado, es recomendable que los criterios de evaluación del portafolio estén a disposición de los alumnos desde el inicio de la ejerci$\operatorname{cio}^{9,18,32,33}$.

En referencia a los resultados de nuestra experiencia, la participación de los alumnos en la realización del portafolio puede considerarse como satisfactoria. En el primer año, a pesar del conflicto que hemos comentado, aproximadamente la mitad de los estudiantes optaron por realizarla. Durante el segundo año la participación fue muy superior y acorde con las expectativas previas de los responsables educativos.

Respecto a los fines pretendidos, queremos resaltar que todos los estudiantes participantes superaron la actividad según el cumplimiento de los requisitos mínimos establecidos. En general los portafolios presentados evidenciaban que los estudiantes habían adquirido un nivel muy considerable en las competencias genéricas evaluadas.

Otro de nuestros objetivos consistía en determinar el tiempo invertido por los participantes en la realización o en la tutorización del portafolio. En los dos cursos estudiados, los estudiantes dedicaron una medica de entre 12 y 13 horas en asumir las distintas tareas de la actividad (preparación de las dos reuniones estudiante-tutor y elaboración del portafolio final). A esta dedicación habría que sumarle el tiempo invertido en las dos reuniones realizadas con el tutor, resultando un tiempo total medio entre 14 y 15 horas de dedicación global al ejercicio por cada estudiante. Así, la realización del portafolio propuesto supondría una dedicación por parte del alumno de un tiempo medio inferior al de un crédito europeo ECTS (el "Sistema Europeo de Transferencia e Créditos" prevé 25 horas por ECTS, en nuestro caso la actividad de 15 horas equivaldrían a 0,6 créditos ECTS). Solamente en algún caso, en los alumnos que dedicaron un mayor tiempo, la actividad fue equivalente a un crédito europeo.

El tiempo medio destinado por los tutores a la actividad, sumando las dos reuniones realizadas con cada alumno, fue de 1,5 a 2,5 horas por estudiante, dedicación que se puede considerar asequible si el tutor tiene a su cargo un número reducido de alumnos.

En referencia a las actitudes mostradas por los implicados, la satisfacción de los estudiantes sobre la actividad fue moderada, si bien más alta en el segundo año. Estos resultados coinciden con los obtenidos en otras experiencias iniciales del portafolio en las que las valoraciones de los participantes al finalizar el ejercicio superaban claramente las expectativas que se habían creado al inicio de la actividad ${ }^{20,34}$. Los tutores por su parte hicieron una valoración muy positiva de la experiencia en ambos años.

A partir de los datos que hemos comentados y de otros procedentes de un estudio más amplio ${ }^{35}$ valoramos muy positivamente la experiencia realizada con los estudiantes del último curso. Esta valoración nos permitió que, en el marco de una experiencia piloto de adaptación de los estudios de Biología de la UPF al EEES impulsada por las autoridades educativas de la "Generalitat" de Cataluña, se generalizara la utilización del portafolio comentado. Las dos innovaciones más importantes de dicha experiencia piloto fueron la introducción del método de aprendizaje basado en problemas (ABP) como parte de la enseñanza de todas las asignaturas y la introducción del portafolio desde el primer curso de la carrera.

Estamos convencidos de que un portafolio de las 
características del que hemos comentado facilitaría el desarrollo de las competencias transversales de los graduados y, como consecuencia, también podría favorecer la inserción laboral de los egresados y su desarrollo profesional futuro.

\section{BIBLIOGRAFÍA}

1. Driessen EW, van Tartwijk J, Vermunt JD, van der Vleuten CPM. Use of portfolios in early undergraduate medical training. Med Teach. 2003; 25: 18-23.

2. Friedman Ben David M, Davis MH, Harden RM, Howie PW, Ker J, Pippard MJ. AMEE Medical Education Guide $\mathrm{N}^{\circ} 24$ : Portfolios as a method of student assessment. Med Teach. 2001; 23: $535-548$

3. Challis M. AMEE Medical Education Guide $\mathrm{N}^{\circ} 11$ (revised): Portfolio-based learning and assessment in medical education. Med Teach. 1999; 21: 370-386.

4. Rees CH. The use (and abuse) of the term "portfolio". Med Educ. 2005; 39: 436

5. Rees CH. "Portfolio" definitions: do we need a wider debate? Med Educ. 2005; 39: 1140-1142.

6. Driessen EW, van Tartwijk J, Overeem K, Vermunt JD, van der Vleuten CPM. Conditions for successful reflective use of portfolios in undergraduate medical education. Med Educ. 2005; 39 : 1230-1235.

7. Royal College of General Practitioners. Occasional Paper 63: Portfolio-based Learning in General Practice. London: The Royal College of General Practitioners, 1993.

8. Van der Vleuten CPM, Schuwirth L. Assessing professional competence: from methods to programmes. Med Educ. 2005; 39: 309-317.

9. Mateos J, Arboix E, Barà J, Ferrer F, Font J, Forns M, Monreal P, Pérez J, Sangrà A. Marc general per a l'avaluació dels aprenentatges dels estudiants. Barcelona: Agència per a la Qualitat del Sistema Universitari a Catalunya; 2003.

10. Davis MH, Friedman Ben-David M, Harden RM, Howie P, Ker J, McGhee C, Pippard MJ, Snadden D. Portfolio assessment in medical students' final examinations. Med Teach. 2001; 23: 357366.

11. Driessen EW, van der Vleuten CPM, Schuwirth L, van Tartwijk J, Vermunt J. The use o qualitative research criteria for portfolio assessment as an alternative to reliability evaluation: a case study. Med Educ. 2005; 39: 214-220.

12. Pérez, J. L'impuls al debat pedagògic: Projecte educatiu de la Facultat de Ciències de la Salut i de la Vida de la Universitat Pompeu Fabra. En: AQU. La implantació de les millores. Barcelona: Agència per a la Qualitat del Sistema Universitari a Catalunya; 2001, p. 129-135.

13. Baños JE, Pérez J. Cómo fomentar las competencias transversales en los estudios de Ciencias de la Salud: una propuesta de actividades. Educ Med. 2005; 8: 216-225.
14. Aramburu J, Bosch F, Pérez J, Sentí M, Baños JE. Los itinerarios profesionales en Biología: un ejemplo de formación académica orientada a la inserción profesional. Educ Med. 2006; 9: 221-228.

15. Valero M, Pérez J. Guia del Portafoli de l'estudiant. Materials Docents Núm. 2. Barcelona: Oficina de Coordinació i Avaluació Acadèmica. Facultat de Ciències de la Salut i de la Vida. Universitat Pompeu Fabra, 2005.

16. Pérez J. Projecte de tutories personals per els estudiants de la Facultat de Ciències de la Salut i de la Vida. Barcelona: Oficina de Coordinació i Avaluació Acadèmica. Facultat de Ciències de la Salut i de la Vida. Universitat Pompeu Fabra, 1999.

17. Davis MH. Portfolios, projects and dissertations. En: Dent JA, Harden RH, editors. A practical guide for medical teachers. London: Harcourt Publishers Limited, 2001, p. 344-356.

18. Challis M. Portfolios and assessment: meeting the challenge. Med Teach. 2001; 23: 437-440.

19. Bordas MI, Cabrera FA. Estrategias de Evaluación de los aprendizajes centrados en el proceso. Rev Española Pedag. 2001; 218: 25-48.

20. O'Sullivan PS, Reckase MD, Mc Clain T, Savidge MA Clardy JA. Demonstration of Portfolios to Assess Competency of Residents. Adv Health Sci Educ. 2004; 9: 309-323.

21. Roberts C, Newble D, O'Rourke A. Portfolio-based assessments in medical education: are they valid and reliable for summative purposes?. Med Educ. 2002; 36: 899-900.

22. Garrett B, Towles E, Kleinert H, Kearns J. Portfolios in LargeScale Alternate Assessment Systems: Framework for Reliability. Assess effective Interv. 2003; 28: 17-27.

23. McMullan M, Endacott R, Gray M, Jasper M, Miller C, Scholes J, Webb C. Portfolios and assessment of competence: a review of the literature. J Adv Nurs. 2003; 41: 283-294.

24. Melville C, Rees M, Brookfield D, Anderson J. Portfolios for assessment of paediatric specialist registars. Med Educ. 2004; 38: 1117-1125.

25. Rolfe I, Mcpherson J. Formative Assessment: How Am I Doing? Lancet. 1995; 345: 837-839.

26. Cohen-Schonatus J. Student assessment and examination rules. Med Teach. 1999; 21: 318-321.

27. Wass V, van der Vleuten CPM, Shatzer J, Jones R. The assessment of clinical competence. Lancet. 2001; 357: 945-949.

28. Dolan G, Fairbairn G, Harris S. Is our student portfolio valued? Nurse Educ Today. 2004; 24: 4-13.

29. Pearson DJ, Heywood P. Portfolio use in general practice vocational training: a survey of GP registrars. Med Educ. 2004; 38 : 87-95.

30. Glen S, Hight NF. Portfolios: An "affective" assessment strategy? Nurse Educ Today. 1992; 12: 416-423.

31. Struyven K, Dochy F, Janssens S. Students' Perceptions about New Modes of Assessment in Higher Education: a Review. En: Segers M, Dochy F, Cascallar E, editors. Optimising New Modes of Assessment: In Search of Qualities and Standards. Dordrecht: Kluwer Academic Publishers, 2003. p. 171-223. 
32. Visser K, Prince K, Sherphbier A, van del Vleuten CPM, Verwijnen G. Students can be full partners in designing their education. Acad Med. 1997; 72: 1034-1035.

33. Hudson JN, Tonkin AL. Evaluating the impact of moving from discipline-based to integrated assessment. Med Educ. 2004; 38 : 832-843.

34. Mathers NJ, Challis MC, Howe AC, Field NJ. Portfolios in con- tinuing medical education - effective and efficient? Med Educ. 1999; 33: 521-530.

35. Valero M. Introducció del portafoli de l'estudiant i del portafoli docent a la Facultat de Ciències de la Salut i de la Vida de la Universitat Pompeu Fabra. Tesis Doctoral. Barcelona: Universitat Pompeu Fabra, 2006. Accesible en: http://www.tesisenxarxa.net/ 